\title{
Speciation Analysis of Butyltin Species in Water by Gas Chromatography with Flame Photometric Detection Using Quartz Surface- induced Tin Emission
}

\author{
G. B. Jiang* and F. Z. Xu \\ Research Centre for Eco-Environmental Sciences, Chinese Academy of Sciences, Beijing 100085, \\ People's Republic of China
}

\begin{abstract}
A flame photometric detector using quartz surfaceinduced tin emission was designed and evaluated for quantification analysis of butyltin species. It has been demonstrated that this quartz surface-induced tin emission, centred at $390 \mathrm{~mm}$, is more sensitive than the commonly used gas-phase emission at $610 \mathrm{~nm}$. The dependence of detector response on quartz enclosure was studied. The operational variables such as hydrogen-air flow rate, carriergas flow rate and purge-gas flow rate were optimized. An analytical procedure for speciation analysis of butyltin species in water using simultaneous hydride generation with sodium borohydride and extraction into dichloromethane was established. The detection limits (defined as the signals that equal three times the deviations of the noise) were $0.3 \mathrm{pg}$ of $\mathrm{Sn}$ for tetrabutyltin (TeBT), $5 \mathrm{pg}$ of Sn for monobutyltin (MBT), $18 \mathrm{pg}$ of $\mathrm{Sn}$ for dibutyltin (DBT) and $2 \mathrm{pg}$ of Sn for tributyltin (TBT), which are approximately 10- to 30 -fold better than those reported for using more commonly used gas-phase emission centred at $610 \mathrm{~nm}$.
\end{abstract}

Keywords: gas chromatography; flame photometric detector; quartz tube; butyltin species; water analysis

\section{INTRODUCTION}

Butyltin species, such as tributyltin (TBT) and its degradation products, dibutyltin (DBT) and monobutyltin (MBT), exhibit toxicological properties and environmental effects. Consequently, it is imperative that analytical techniques used for

\footnotetext{
* Author to whom all correspondence should be addressed.
}

butyltin analysis are able to differentiate the various species at ultra-trace levels. A variety of analytical methodologies have been developed over the last 15 years. Most techniques, however, are based on the selective determination of tin compounds in complex matrices such as atomic absorption spectrometry, ${ }^{1-4}$ gas or liquid chromatography, ${ }^{5,6}$ electron impact or ion-spray mass spectrometry 7,8 and inductively coupled plasma-atomic emission spectrometry. ${ }^{9}$ To differentiate butyltin species, various gas or liquid chromatographic modes must be combined with selective detection methods to produce hyphenated techniques; these mainly include gas or liquid chromatography with atomic absorption spectrometry, ${ }^{10-14}$ gas chromatography with electron capture detection ${ }^{15,16}$ and gas chromatography with flame photometric detection. ${ }^{17-19}$

Gas chromatography with flame photometric detection (GC-FPD) is often the analytical method of choice because of its high separation ability, high sensitivity and also general laboratory availability. In GC-FPD, the detection is based on the tin molecular emission in a hydrogen-air flame. The band most commonly selected is centered at $610 \mathrm{~nm}$ and is attributed to $\mathrm{SnH}$ emission in the gas phase. ${ }^{20} \mathrm{~A}$ broader emission band, 100-1000 times more sensitive, centred at about $390 \mathrm{~nm}$ and attributed to a quartz surface-induced tin emission, was observed later by Aue and his co-workers. ${ }^{21,22}$ However, despite its great advantages in sensitivity, this model of emission has not actually been employed for tin determination, while the less sensitive gasphase emission is extensively used. The possible reason is its temperamental nature and susceptibility to poisoning.

During our previous studies, ${ }^{23}$ a laboratorymade flame photometric detector was used, which demonstrated, for the first time that this blue tin 
emission model is sufficiently stable, reproducible and rugged to be used in the quantificative determination of environmental samples. The present study is designed to investigate further the detector performance and to optimize the experimental parameters. Particular attention is devoted to establishing an ultra-trace method for speciation analysis of butyltin species in water using a simultaneous hydride generation procedure.

\section{EXPERIMENTAL}

\section{Instrumentation}

A flame photometric detector of our own design with a similar configuration to that described in a previous report ${ }^{23}$ was constructed. It was a combination of a flame burner and an optical system. The burner used three deactivated, coaxially silver-soldered, stainless steel tubes. The burner position was designed to be easily adjusted up and down without affecting the operation of the optical system. The column effluent was brought directly through the innermost tube to the burner nozzle and was not premixed with hydrogen and air. To obtain better flame stability and high sensitivity, hydrogen was fed into the outer tube while air was introduced to the middle tube between the innermost and outer tube. The hydrogen-rich flame burned on the top of the burner nozzle, shielded by a $7 \mathrm{~mm}$ i.d. $\times 70 \mathrm{~mm}$ straight quartz tube, which provided a clean inner surface to the blue tin emission. A Shimadzu GC-6A gas chromatograph-based FPD electronic system was used with a newly designed flame photometric detector along with a conventional, sensitive photometric tube (Hamamatsu TV Co., Ltd; R-268) and a $394 \mathrm{~nm}$ interference filter. The output signal was recorded by a strip chart recorder in the peak height mode (Shimadzu, Model R-12).

The detector was mounted at the front on top of a gas chromatograph (Shimadzu; Model GC$6 \mathrm{~A})$. A $2 \mathrm{~m} \times 3 \mathrm{~mm}$ i.d. borosilicate glass column packed with $1.5 \%$ OV-101 on Chromosorb G, $80 /$ 100 mesh, was used throughout the experiment. The transfer tube between chromatographic oven and detector was kept at least $20^{\circ} \mathrm{C}$ higher than the maximum column temperature. The optimized chromatographic conditions were: injector port, $200{ }^{\circ} \mathrm{C}$; detector body, $180^{\circ} \mathrm{C}$; transfer tube,
$200{ }^{\circ} \mathrm{C}$; carrier gas (nitrogen), $40 \mathrm{~cm}^{3} \mathrm{~min}^{-1}$, which was further purified by sequentially passing through a molecular-sieve $5 \AA$ trap. Typical flow rates of the detector gases were: air, $100 \mathrm{~cm}^{3} \mathrm{~min}^{-1}$; and hydrogen, $200 \mathrm{~cm}^{3} \mathrm{~min}^{-1}$. The column temperature programme was as follows: initially, $50{ }^{\circ} \mathrm{C}$ for $2 \mathrm{~min}$; ramp at $8{ }^{\circ} \mathrm{C}$ min $^{-1}$; and finally $200{ }^{\circ} \mathrm{C}$.

\section{Reagents}

Tributyltin chloride $(98.6 \%)$, monobutyltin chloride $(99 \%)$ and tetrabutyltin $(99.4 \%)$ were obtained from M\&T Chemical Inc., USA; bis(dibutyltin)oxide $(96 \%)$ was obtained from Ciba-Geigy. Each butyltin compound was dissolved in methanol at a concentration of $1 \mathrm{mg} \mathrm{Sn} \mathrm{cm}^{-3}$ as a stock solution and refrigerated when not in use. Working standard solutions were prepared by diluting stock solutions with methanol or distilled water before use.

\section{Hydride generation}

Ionic organometallic compounds such as MBT, DBT and TBT species generally have rather high boiling points so a derivatization step is required to lower their boiling points to appropriate values for GC analysis. Hydride generation and alkylation are two major and powerful techniques in that derivatization process. For aqueous samples, hydride generation has an advantage over alkylation. Hydride generation produces a large volume of hydrogen as a byproduct that facilitates the purging of butyltin hydrides. Simultaneous hydride generation and extraction into dichloromethane for GC-FPD analysis has been described by Matthias et al.;8 however, this procedure required a large volume of sample and extraction twice with dichloromethane; the extract needs to be concentrated to $50-100 \mu \mathrm{l}$ with careful treatment. In this case, it is rather tedious and timeconsuming. Due to the high sensitivity available for the quartz surface-induced tin emission, the procedure we propose here is quite simple; no evaporation concentration step was required, even when the concentration of sample is below the $1 \mathrm{ng} \mathrm{cm}^{-3}$ level.

\section{Analysis procedure}

For a typical analysis of water, a $20 \mathrm{~cm}^{3}$ sample was placed in a $50 \mathrm{~cm}^{3}$ glass separatory funnel; $2 \mathrm{~cm}^{3}$ of dichloromethane was added, followed by 
$1 \mathrm{~cm}^{3}$ of $4 \%(\mathrm{w} / \mathrm{v})$ aqueous $\mathrm{NaBH}_{4}$. The funnel was capped and gently shaken by hand for $1 \mathrm{~min}$, vented, and then shaken on an electric shaker (Beijing Kangshi Machine Factory) at 245 strokes $\min ^{-1}$ for $15 \mathrm{~min}$. After $5 \mathrm{~min}$ settling time, the lower organic layer was removed to a $1 \mathrm{~cm}^{3}$ capped glass vial which was chilled on ice; $1 \mu \mathrm{l}$ of this solution was required for $\mathrm{GC}$ analysis.

\section{RESULTS AND DISCUSSION}

\section{Quartz enclosure}

Previous studies indicated that tin emission, which occurs at the clean quartz surface, can be obtained with differently shaped quartz tube, ${ }^{21,22}$ or even by use of quartz wool. ${ }^{24}$ As a consequence of our experiments, the intense blue tin emission could be easily observed for various butyltin species with differently shaped quartz tubes; however, the response and the peak shape changed drastically when the quartz tube was altered. By a series of tests, we found that a straight quartz tube, giving satisfactory sensitivity, reproducibility and stability, is the most suitable choice for quantification purposes. Quartz tubes having other shapes, even those giving much better sensitivity, cannot perform so well under the same conditions as peak broadening, asymmetry and lower reproducibility limit their quantitative utility.

The dimension of the straight quartz tube is the major factor for improving peak broadening and for increasing detector response. It was proved that peak broadening can be reduced by decreasing the diameter of the tube, but a flame-out problem caused by small-diameter quartz tubes should also be considered. The suitable diameter of the quartz tube for our detector is $7 \mathrm{~mm}$ i.d. and $70 \mathrm{~mm}$ in height, which is about $2 \mathrm{~mm}$ bigger in diameter than the burner. After prolonged use, the quartz surface coud be 'poisoned', diminishing the detector sensitivity, possibly due to reactions between the inner wall of the quartz tube and the hydrogen flame at high temperature. Injection of fluorine-containing compounds, a method which has been commonly used to remove silica deposits from detector, ${ }^{21,} 22$ showed no significant improvement on detector response. A practical aid is to inject $2-5 \mu \mathrm{l}$ of pure methanol (up to 10 times) through the column; this produced strong effects in the flame region and hence cleaned the quartz surface to a certain extent. If the response drops

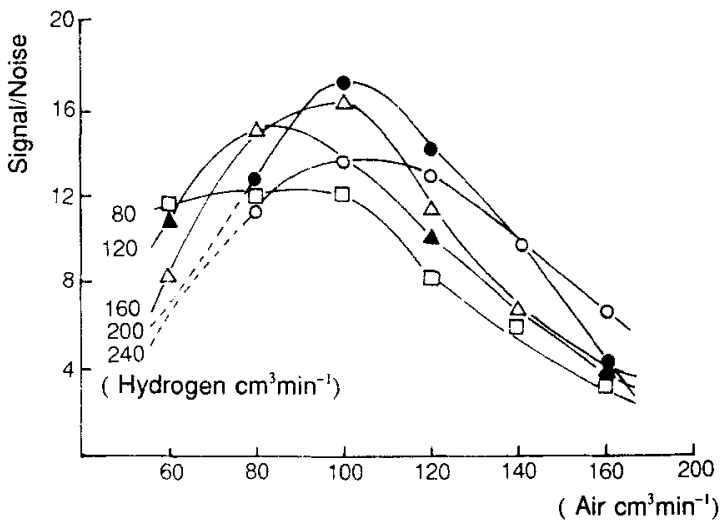

Figure 1 Detector response versus various air and hydrogen flow rates; $0.02 \mathrm{ng}$ of TeBT was injected. Oven temperature was maintained constaint at $180^{\circ} \mathrm{C}$.

noticeably, the tube should be treated by putting it into aqua regia overnight or by changing to a new tube.

\section{Influence of gas flow rate}

Gas flow rate is one of the most important parameters related to detector performance. To optimize the gas flow rate, $0.02 \mathrm{ng}$ of tetrabutyltin (TeBT) was injected at different hydrogen and air flow rates. The dependence of the detector response for TeBT on hydrogen-air flow rate is shown in Fig. 1. In this experiment, the hydrogen was maintained at flow rates of $80,120,160,200$ and $240 \mathrm{~cm}^{3} \mathrm{~min}^{-1}$, respectively, and the air flow rate was changed from 60 to $160 \mathrm{~cm}^{3} \mathrm{~min}^{-1}$. As shown, for each hydrogen flow rate, there is a

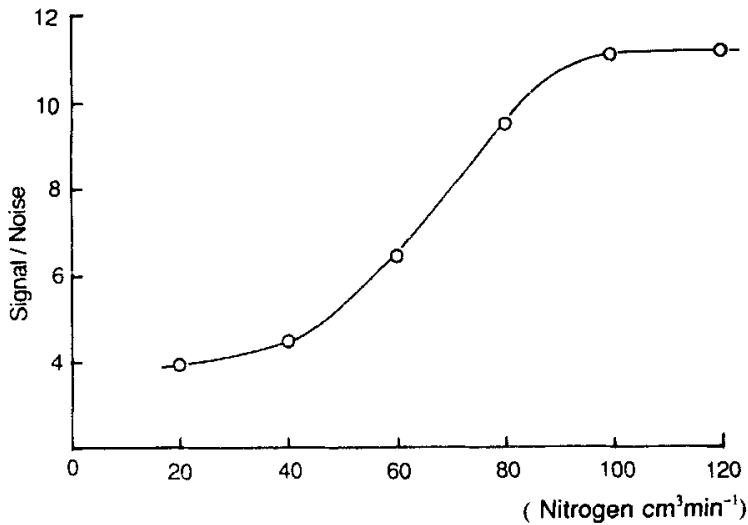

Figure 2 Effect of purge gas on detector response; $0.02 \mathrm{ng}$ of TeBT was injected. Oven temperature was maintained constant at $180^{\circ} \mathrm{C}$ 


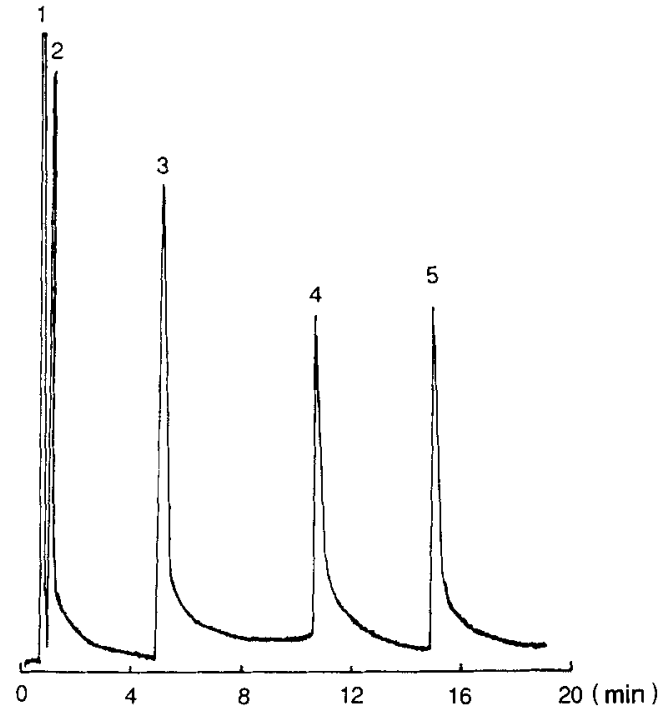

Figure 3 A typical GC-FPD chromatogram for a mixture of four standard butyltin species under optimized GC conditions. Peak 1, solvent; peak 2, $0.5 \mathrm{ng}$ of $\mathrm{Sn}$ as MBT; peak 3, $1 \mathrm{ng}$ of $\mathrm{Sn}$ as DBT; peak 4, 0.5 ng of $\mathrm{Sn}$ as TBT; and peak 5, $0.1 \mathrm{ng}$ of $\mathrm{Sn}$ as TeBT. See text for chromatographic conditions and hydride generation/extraction procedure.

distinct optimum air flow rate. At these optimum values, the hydrogen-to-oxygen ratio is approximately $8: 10$, which is slightly higher than the values generally reported in the literature. ${ }^{25}$ It was noted that changing the flow rate of carrier gas (nitrogen) from 10 to $60 \mathrm{~cm}^{3} \mathrm{~min}^{-1}$ did not appear to cause significant changes in peak height or noise level. However, higher flow rate resulted in an incomplete separation between solvent peak and MBT; therefore, the flow rate was kept at $40 \mathrm{~cm}^{3} \mathrm{~min}^{-1}$.

To solve the flame-out problem caused by water condensing on the detector chimney and to reduce the quenching effects possibly resulting from solvent, hydrocarbons, sulphur and stationary phase, we tried to use nitrogen as purge gas with the output of the transfer tube placed near the vent chimney of the detector housing. It was occasionally found that the purge gas had a strong enhancement effect on detector response. Figure 2 indicates that when the nitrogen purge-gas flow rate changed from 60 to $120 \mathrm{~cm}^{3} \mathrm{~min}^{-1}$, two- to three-fold increase in detector response was obtained. Some of this can be attributed to the added purge gas acting as an inert gas having the effect of lowering the temperature of the hydrogen-air flame, removing the interference and keeping the detector housing dry.

\section{GC of butyltin species}

Figure 3 shows a typical chromatogram of a $1 \mu \mathrm{l}$ injection of a mixed standard butyltin hydrides solution containing $0.5 \mathrm{ng}$ of $\mathrm{Sn}$ as MBT and TBT, $1 \mathrm{ng}$ of $\mathrm{Sn}$ as DBT, and $0.1 \mathrm{ng}$ of $\mathrm{Sn}$ as TeBT. Since the chromatographic column was packed with $1.5 \%$ OV-101 on Chromosorb G (80100 mesh), the chromatographic peak, as is often the case with a packed column, shows some tailing, but it did not interfere with the quantification analysis. A few per cent (less than $2 \%$ ) of DBT may rearrange to MBT and TBT during column separation, and this is one reason for the recovery error.

\section{Response curves and detection limits}

To prepare the response curve, a standard solution was made by mixing different amounts of MBT $\quad\left(0.1-0.5 \mu \mathrm{g} \mathrm{cm}^{-3}\right)$, DBT $\quad(0.2-$ $1.0 \mu \mathrm{g} \mathrm{cm}^{-3}$, TBT $\left(0.1-0.5 \mu \mathrm{g} \mathrm{cm}^{-3}\right)$ and TeBT $\left(0.02-0.10 \mu \mathrm{g} \mathrm{cm}^{-3}\right.$ in a $50 \mathrm{~cm}^{3}$ glass separatory funnel, diluted with $20 \mathrm{~cm}^{3}$ distilled water, to which was added $2 \mathrm{~cm}^{3}$ dichloromethane, followed by hydride generation which was accomplished using the procedure given above. The response curves were made by plotting peak heights versus the amount of standard solution injected. The quality of the determination was evaluated by preparing a response curve each week and injecting a hydride-generated standard solution with all four butyltin compounds every

Table 1 Response curve data for butyltin species

\begin{tabular}{llllll}
\hline Species & $\begin{array}{l}\text { Concentration } \\
\text { range (ng, as Sn) }\end{array}$ & Slope, $m$ & $Y$-intercept & $\begin{array}{l}\text { Correlation } \\
\text { coefficient }\end{array}$ & $\begin{array}{l}\text { Detection } \\
\text { limit (pg, as Sn) }\end{array}$ \\
\hline MBT & $0.1-0.5$ & 25.8 & -0.16 & 0.9992 & 5 \\
DBT & $0.2-1.0$ & 10.15 & 0.05 & 0.9874 & 18 \\
TBT & $0.1-0.5$ & 15.5 & 0.11 & 0.9994 & 2 \\
TeBT & $0.02-0.10$ & 85.0 & 0.10 & 0.9989 & 0.3 \\
\hline
\end{tabular}


Table 2 Recovery of butyltin species (\%) from spiked water samples $^{a}$

\begin{tabular}{lrrrr}
\hline Sample & \multicolumn{1}{c}{ MBT } & \multicolumn{1}{c}{ DBT } & \multicolumn{1}{l}{ TBT } & \multicolumn{1}{c}{ TeBT } \\
\hline Waste water & $85.5 \pm 3.1$ & $101.2 \pm 4.8$ & $111.3 \pm 5.9$ & $86.9 \pm 3.6$ \\
River water & $106.3 \pm 3.6$ & $102.4 \pm 4.1$ & $106.8 \pm 3.6$ & $101.3 \pm 3.0$ \\
Dalian Bay & $101.2 \pm 6.4$ & $90.8 \pm 7.7$ & $89.0 \pm 7.2$ & $93.2 \pm 4.1$ \\
Estuarine & $91.4 \pm 3.4$ & $84.1 \pm 4.1$ & $86.7 \pm 2.5$ & $87.8 \pm 2.6$ \\
water & & & & \\
\hline
\end{tabular}

a Spiked with $0.1,0.2,0.1$ and $0.01 \mu \mathrm{g}$ of Sn for MBT, DBT, TBT and TeBT, respectively, in a $20 \mathrm{ml}$ sample. There was no detectable amount of butyltin species in unspiked samples. Results given are averages of independent duplicate analyses.

day to check the detection reproducibility. The regression equations (see Table 1) show good linearity within the concentration ranges studied but different response factors with respect to each species. In addition, a good instrumental linear range for TeBT, over four orders of magnitude, from 0.002 to $20 \mathrm{ng}$ with a correlation of 0.9970 , was observed. The absolute detection limits, calculated as three times the standard deviation of the noise, are also listed in Table 1 . Since only a $20 \mathrm{~cm}^{3}$ sample volume was used for water analysis, this placed the relative detection limits between 0.04 and $0.102 \mathrm{ng} \mathrm{cm}^{-3}$, depending upon the different butyltin species. A further increase in relative detection limits is also easy to realize by using a larger volume of sample.

\section{Recovery and precision}

Of all the extractants tested for the extraction of butyltin species from water samples, dichloromethane is effective for butyltin hydrides. The extraction efficiency for butyltin species from water samples was investigated by spiking known amounts of the compounds into $20 \mathrm{~cm}^{3}$ samples and performing the simultaneous hydride generation/extraction procedure. The average recoveries of the four compounds ranged from 84.1 to
$106.8 \%$ (see Table 2), which was similar to the recoveries of $84-114 \%$ obtained by the ethylation procedure ${ }^{14}$ and was better than the recoveries of $70-95 \%$ using the benzene extraction/hydride generation procedure. ${ }^{16}$ The precision of the method was assessed by running replicate $(n=9)$ analyses of a waste-water sample, which was diluted 25 -fold with distilled water. The relative standard deviations were $2.08 \%$ for MBT and $2.78 \%$ for DBT. No detectable amounts of TBT and TeBT were found in this sample.

\section{Analysis of butyltin species in water}

The procedure based on the sensitive quartz surface-induced tin emission mode was applied to the determination of butyltin species in water samples. The results are given in Table 3 . River and lake water were freshly collected from the urban area of Beijing at $10 \mathrm{~cm}$ below the water surface. For marine samples, $1 \mathrm{~cm}^{3}$ of concentrated hydrochloric acid per litre of sample was added after collection. The bottles were placed in a cooling container during transportation.

\section{CONCLUSIONS}

A flame photometric detector using quartz surface-induced tin emission with simultaneous extraction and hydride generation procedure has been shown sufficiently stable, sensitive and rugged to be used in quantification analysis of butyltin species in water samples. Further study will apply the presented detector to various biological sample and sediment determinations. The use of a capillary column or megabore column seems to be a prerequisite to avoid peak tailing and to minimize degradation or rearrangement problems during the chromatographic

Table 3 Concentrations of butyltin species $\left(\mu \mathrm{g} \mathrm{Sn} \mathrm{dm}^{-1}\right)$ in water ${ }^{a}$

\begin{tabular}{lllll}
\hline Sample & MBT & DBT & TBT & TeBT \\
\hline Waste water & & & & \\
$(1)$ & $0.85 \pm 0.02$ & $2.59 \pm 0.11$ & $0.162 \pm 0.006$ & $0.013 \pm 0.001$ \\
$(2)$ & $12.71 \pm 0.46$ & $2.58 \pm 0.15$ & $1.41 \pm 0.11$ & $0.143 \pm 0.012$ \\
Marine water & $0.026 \pm 0.003$ & nd & nd & nd \\
Lake water & nd & nd & nd & nd \\
River water & nd & nd & nd & nd \\
\hline
\end{tabular}

${ }^{a}$ Means of triple analyses. nd Not detected. 
process. Such improvement is under way in our present studies.

Acknowledgement This work was financially supported by the National Natural Science Foundation of China. G. B. J. thanks Dr K. W. M. Siu, Institute for Environmental Chemistry, National Research Council of Canada, for his valuable suggestions during the previous work.

\section{REFERENCES}

1. P. N. Vijan and C. Y. Chan, Anal. Chem. 48, 1788 (1976).

2. V. F. Hodge, S. L. Seidel and E. D. Goldberg, Anal. Chem. 51, 1256 (1979).

3. M. O. Andreae and J. T. Byrd, Anal. Chim. Acta 156, 147 (1984).

4. O. F. X. Donard, S. Rapsomanikis and J. H. Weber, Anal. Chem. 58, 772 (1986).

5. W. O. Gauer, J. N. Seiber and D. G. Grosby, J. Agric Food Chem. 22, 252 (1974).

6. R. S. Braman and M. A. Tompkins, Anal. Chem. 51, 12 (1979).

7. H. A. Meinema, T. Burger-Wiersma, G. Versluis-de Haan and E. C. Gevers, Environ. Sci. Technol. 12, 288 (1978).

8. K. W. M. Siu, G. J. Gardner and S. S. Berman, Anal. Chem. 61, 2320 (1989)

9. H. Suyani, J. Creed, T. Davidson and J. Caruso, J. Chromatogr. 209, 139 (1989).
10. F. E. Brinckman, W. R. Blair, K. L. Jewett and W. P. Iverson, J. Chromatogr. Sci. 15, 493 (1977).

11. L. Ebdon, S. J. Hill and P. Jones, Analyst (London) 110 , 515 (1985).

12. O. Nygren and C.-A. Nilson, Spectrochim. Acta 44B, 589 (1989).

13. Y. K. Chau, P. T. S. Wong and G. A. Bengert, Anal. Chem. 54,246 (1982).

14. Y. K. Chau, S. Z. Zhang and R. J. Maguire, Analyst (London) 117, 1161 (1992).

15. Y. Arrakawa, O. Wada, T. H. Hu and $H$. Iwai, J. Chromatogr 216, 209 (1981).

16. Y. Hattori, A. Kobayashi, S. Takemoto, K. Takami, Y. Kuge, A. Sugimae and M. Nakamoto, J. Chromatogr. 215, 341 (1984).

17. R. J. Maguire and H. Huneault, J. Chromatogr. 209, 458 (1981).

18. C. L. Matthias, J. M. Bellama, G. J. Olson and F. E. Brinckman, Environ. Sci. Technol. 20, 609 (1986).

19. A. O. Valkirs, P. F. Seligman, C. L. Matthias and J. M. Bellama, Analyst (London) 112, 17 (1987).

20. S. S. Brody and J. E. Chaney, J. Gas Chromatogr. 4, 42 (1966).

21. W. A. Aue and C. G. Flinn, J. Chromatogr. 142, 145 (1977).

22. C. G. Flinn and W. A. Aue, J. Chromatogr. 186, 299 (1979).

23. G. B. Jiang, P. S. Maxwell, K. W. M. Siu, V. T. Luong and S. S. Berman, Anal. Chem. 63, 1506 (1991).

24. W. A. Aue and C. G. Flinn, Anal Chem. 52, 1537 (1980).

25. C. A. Burgett and L. E. Green, J. Chromatogr. 12, 356 (1974). 